\title{
CORRELACIÓN ENTRE EL PESO ESTIMADO POR ECOGRAFÍA Y EL PESO DEL RECIÉN NACIDO EN GESTANTES A TERMINO DEL CENTRO DE SALUD LA ESPERANZA - TACNA 2016
}

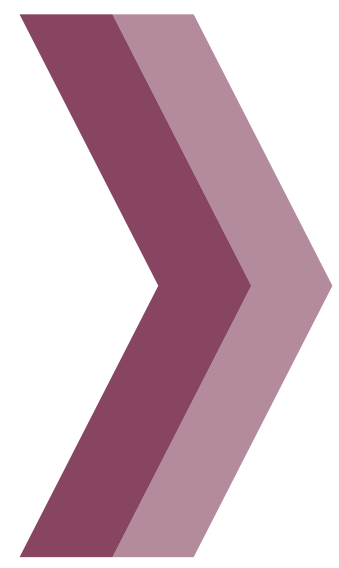

\section{CORRELATION BETWEEN THE WEIGHT ESTIMATED BY \\ ECOGRAPHY AND THE WEIGHT OF THE NEWLY BORN IN GESTANTS OF THE HEALTH CENTER LA ESPERANZA - TACNA 2016}

\section{RESUMEN}

INTRODUCCIÓN: La ecografía obstétrica es fundamental para identificar posibles riesgos para la madre y el niño; además de estimar el peso del recién nacido, nos ayuda a determinar la vía del parto. El objetivo fue determinar la correlación entre el peso fetal estimado por ecografía y peso del recién nacido. MATERIAL Y MÉTODOS: Estudio descriptivo correlacional, retrospectivo y de corte transversal. La muestra estuvo constituida por 129 gestantes,a término con feto único de 37 a 41 semanas, con bajo riesgo obstétrico y con ecografía entre las 37 a 41 semanas; cuya atención de parto fue en el Centro de Salud La Esperanza MINSA de la Región Tacna, durante el año 2016. La técnica utilizada fue el análisis documental de las historias clínicas y libro de partos. El instrumento fue una ficha de recolección de datos y su análisis mediante frecuencias y porcentajes y chi2 además de pruebas correlaciónales de Pearson. RESULTADOS: Las gestantes presentaron edades entre los 18 a 29 años $(64,3 \%)$, con secundaria completa $(63,6 \%)$, talla de 1,50 a $1,60 \mathrm{~cm}(62,8 \%)$ y peso materno de 64 a $76 \mathrm{~kg}$ $(66,6 \%)$, secundíparas $(38,8 \%)$ con edad gestacional de 39 y 40 semanas por ecografía $(42,6 \%$ y $27,1 \%)$. El peso fetal determinado por ecografía fueron $73,6 \%$ pesos normales, $24 \%$ alto pesoy $2,3 \%$ bajo peso. Con respecto al peso de nacimiento presentaron $65,1 \%$ pesos normales; $27,9 \%$ alto peso; $4,7 \%$ macrosómicos y $2,3 \%$ con bajo peso. CONCLUSIÓN: Existe relación significativa entre el peso fetal estimado por ecografía y el peso al nacimiento $(p<0,01)$.

PALABRAS CLAVE: Peso fetal estimado por ecografía, peso observado al nacer, gestantes a término.

\section{Luis Fredy Choque Mamani ${ }^{1}$}

1. Obstetra del Centro de Salud La Esperanza MINSA Tacna. Docente de la Facultad Ciencias de la Salud. Universidad Nacional Jorge Basadre GrohmannTacna

\section{ABSTRACT}

INTRODUCTION: Obstetric ultrasound is essential to identify possible risks for the mother and the child; In addition to estimating the weight of the newborn, it helps us determine the way of delivery. The objective was to determine the correlation between estimated fetal weight by ultrasound and newborn weight. MATERIAL AND METHODS: Descriptive, correlational, retrospective and cross-sectional study. The sample consisted of 129 pregnant women, at term with a single fetus from 37 to 41 weeks, with low obstetric risk and with ultrasound between 37 to 41 weeks whose delivery care was at the Health Center La Esperanza MINSA of the Tacna Region, during the year 2016. The technique used was the documental analysis of the medical records and birth book. The instrument was a data collection card and its analysis by frequencies and percentages and chi 2 in addition to Pearson correlation tests. RESULTS: Pregnant women had ages between 18 and 29 years old $(64.3 \%)$, with complete secondary education (63.6\%), height from 1.50 to $1.60 \mathrm{~cm}$ $(62.8 \%)$ and maternal weight of 64 . to $76 \mathrm{~kg}(66.6 \%)$, secundiparous (38.8\%) with gestational age of 39 and 40 weeks by ultrasound ( $42.6 \%$ and $27.1 \%)$. The fetal weight determined by ultrasound were $73.6 \%$ normal weight, $24 \%$ high weight and $2.3 \%$ low weight. With respect to the weight of birth they presented $65.1 \%$ normal weights, $27.9 \%$ high weight, $4.7 \%$ macrosomic and $2.3 \%$ with low weight. CONCLUSION: There is a significant relationship between estimated fetal weight by ultrasound and birth weight $(p<0.01)$.

KEYWORDS: Acute pancreatitis, modified Marshall score. 


\section{INTRODUCCIÓN}

La estimación del peso fetal ha sido motivo de diferentes tipos de controles, todos apuntando a una manera de evaluar la salud fetal. El año 1958 se empleó por vez primera el ultrasonido en Obstetricia y Ginecología. A partir de esta publicación las informaciones aparecidas en la literatura demuestran la creciente importancia que se le ha prestado al tema (1). El diagnóstico mediante examen ultrasonográfico se puede emplear en una variedad de circunstancias específicas durante el embarazo, como después de complicaciones clínicas, o donde se considere que el feto presente factores de riesgo importantes (2). La ecografía obstétrica es fundamental para identificar posibles riesgos para la madre y el niño; además hace que el parto sea más seguro para algunas mujeres porque permite controlar la hemorragia postparto, señaló Pablo Jiménez, asesor regional de la OPS/OMS (3). Para el cálculo del peso fetal existen muchas fórmulas que se basan en la medición de la biometría fetal. La primera, publicada por Warsof y Shepard, que utilizaron el diámetro biparietal (DBP) y perímetro abdominal (PA), más tarde Hadlock incorpora la longitud del fémur (LF) y sustituye el diámetro biparietal (DBP) por la circunferencia cefálica (CC), eliminando los errores atribuibles a variaciones de la morfología de la cabeza fetal, obteniendo así una mejor predicción del peso fetal, siendo esta última actualmente la más utilizada a nivel mundial (4).

Díaz (11) en la Unidad de Perinatología de la Universidad de Carabobo, Venezuela, en el año 2009 concluye que la estimación del peso fetal ecográfico es una medida confiable para predecir el peso al nacer, si esta se realiza en los 15 días previos al parto.

En 2013 Teva (12) en Chile, concluye que la ecografía es el estándar dorado para la estimación del peso y la valoración de su crecimiento. Encontró que el error entre el peso real y el estimado no es inferior a 7,5-10\%, y en el caso de recién nacidos macrosómicos puede llegar al $15 \%$.

Ferreiro (13) en el hospital "Ramón González Coro" de Cuba en 2007 demostró que la fórmula de Campbell fue la de mayor sensibilidad y valores predictivos positivo y negativo, pero resultó la de menor especificidad comparada con Hadlock, para estimación de peso fetal por ultrasonido.

Villafuerte (14) en el hospital Nacional Guillermo Almenara de Lima Perú en 2011, afirma que uno de los factores que pueden influenciar en la exactitud de la estimación ecográfica del peso fetal es la experiencia del ecografista, así en los médicos residentes de menor experiencia ( $<6$ meses) $49,4 \%$ de sus estimados ecográficos estuvieron dentro del $10 \%$ del peso real, mientras que en los más experimentados (>24 meses) fue de $73,6 \%$.

Rodríguez (16) en el Hospital Regional de Cajamarca, Perú en 2014 concluye que el ponderado fetal estimado por el método de Johnson-Toshach fue más exacto que la ultrasonografía en gestantes entre $37 \mathrm{y}$ 41 semanas de gestación, para fetos con peso entre 2 501 y $3999 \mathrm{~g}$.

Manuelo (19) en el hospital Hipólito Unanue de Tacna 2009 , concluye que los modelos que estiman el peso fetal por ecografía subestiman el peso al nacer de los recién nacidos a término. El modelo que presenta menor error absoluto, menor error porcentual y mejor correlación positiva con el peso al nacer es el modelo de Hadlock.

El objetivo de este estudio fue determinar la correlación entre el peso fetal estimado por ecografía y peso al nacer del recién nacido en el Centro de Salud La Esperanza Tacna.

\section{MATERIAL Y MÉTODOS}

El estudio es retrospectivo, transversal y no experimental en su modalidad correlacional. Se realizó en el Centro de Salud La Esperanza del MINSA Región Tacna, con datos registrados durante todo el año 2016. La muestra estuvo constituida por 129 gestantes, a término con feto único de 37 a 41 semanas, con bajo riesgo obstétrico y con ecografía entre las 37 a 41 semanas cuya atención de parto fue en este establecimiento de salud. La técnica utilizada fue el análisis documental de las historias clínicas y libro de partos. El instrumento fue una ficha de recolección de datos y su análisis mediante frecuencias y porcentajes y Chi2 además de pruebas correlacionales de Pearson. La edad gestacional se basó en la fecha de última menstruación si esta es conocida y confiable. 


\section{RESULTADOS}

\begin{tabular}{|c|c|c|}
\hline Edad & Frecuencia & $\%$ \\
\hline $\begin{array}{c}\text { Adolescente (12 a } \\
17 \text { Años) }\end{array}$ & 1 & $0,8 \%$ \\
\hline $\begin{array}{c}\text { Joven (18 a } \\
29 \text { Años) }\end{array}$ & 83 & $64,3 \%$ \\
\hline $\begin{array}{c}\text { Adulto (30 a } \\
45 \text { Años) }\end{array}$ & 45 & $34,9 \%$ \\
\hline Total & 129 & $100,0 \%$ \\
\hline
\end{tabular}

En el Centro de Salud La Esperanza, el 64,3\% de las gestantes atendidas se encuentran en la edad joven entre (18 a 29 años); el 34,9\% están en edad adulto (30 a 45 años); y solo el 0,8\% están en edad adolescente (12 a 17 años). Tabla 1.

\section{TABLA № 2}

Distribución de gestantes Según Peso Promedio Ponderado Fetal por Ecografía.

\begin{tabular}{|c|c|c|}
\hline $\begin{array}{l}\text { Promedio Ponderado Fetal } \\
\text { por Ecografía }\end{array}$ & $\begin{array}{l}\text { Frecue } \\
\text { ncia }\end{array}$ & $\begin{array}{r}\text { Porce } \\
\text { ntaje }\end{array}$ \\
\hline Bajo Peso (< a 2500) & 03 & $2,3 \%$ \\
\hline Normo peso ( 2500 a 3500 ) & 95 & $73,6 \%$ \\
\hline Sobre Peso (3501 a 4000) & 31 & $24,0 \%$ \\
\hline Macrosómico ( > de 4000) & 00 & $0,0 \%$ \\
\hline Total & 129 & $100,0 \%$ \\
\hline
\end{tabular}

Reúnen los indicadores según peso promedio ponderado fetal por ecografía, el $73,6 \%$ se observa, que tiene reuniendo, el $24,0 \%$ se observa, que tiene sobrepeso y finalmente el $2,3 \%$ se observa que tiene bajo peso. No se consideró ningún macrosómico por ecografía en nuestro estudio 0,0\%.Tabla 2.

\section{TABLA No 3}

Según Peso del Recién Nacido.

\begin{tabular}{|lcl|}
\hline Peso del Recién Nacido & Nro. & $\%$ \\
\hline Bajo Peso (< a 2500gr.) & 03 & $2,3 \%$ \\
\hline Normopeso( 2500 a 3500 gr.) & 73 & $56,6 \%$ \\
Sobre Peso (3501 a 4000 gr.) & 46 & $35,7 \%$ \\
Macrosómico ( > 4000 gr. ) & 07 & $5,4 \%$ \\
\hline \multicolumn{1}{c}{ Total } & 129 & $100,0 \%$ \\
\hline
\end{tabular}

Reuniendo los indicadores según peso real del recién nacido, tomado inmediatamente después del expulsivo observamos que el $56.6 \%$ nació normo peso (2500 a 3500gr.); el 35,7\% tuvo sobre peso (3501 a 4000 gr.); el 5,4\% fue macrosómico (> a 4000 gr.), y finalmente solo el 2,3\% nació con bajo peso $(<2500$ gr.).

\section{TABLA № 4}

Relación entre edad gestacional estimado por ecografía y por fecha de última menstruación

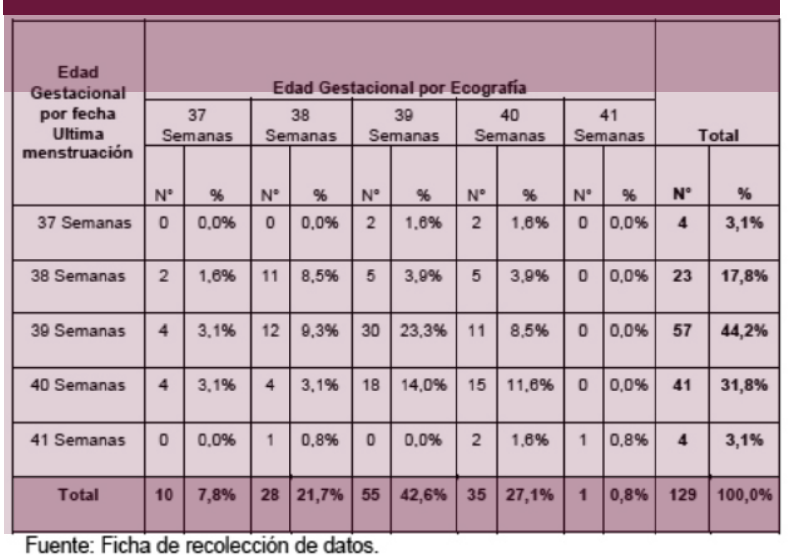

Chi=52,866 $\quad g=16 \quad P=0,00000<a=0,05$

\begin{tabular}{|l|r|r|r|}
\hline & Pruebas de chi-cuadrado \\
\hline & \multicolumn{1}{|c|}{ Valor } & \multicolumn{1}{|c|}{ gl } & Sig. asintótica (bilateral) \\
\hline Chi-cuadrado de Pearson & $52,800^{\circ}$ & 18 & 0,000 \\
Razón de verosimiltudes & 30,723 & 18 & 0,015 \\
Asociación lineal por lineal & 3,528 & 1 & 0,060 \\
N de casos válidos & 128 & & \\
\hline
\end{tabular}

a. 17 casillas $(68,0 \%)$ tenen una frecuencia esperada inferior a 5 . La frecuencia minima esperada es , 03.

Se presentan la relación entre la edad gestacional estimado por ecografía y fecha de última menstruación y se puede observar que a las 39 semanas el $23.3 \%$ de los cálculos estimados han coincidido, seguido del $11,6 \%$ a los 40 semanas, el 9,3 $\%$ a los 38 semanas. Con las pruebas de correlación de Pearson; se tiene que $\mathrm{Chi}=52,866 \mathrm{gl}=16 \mathrm{P}=0,00000$ $<\alpha=0,05$. Entonces la edad gestacional calculada por ecografía y por fecha de ultima menstruación en las gestantes del Centro de Salud La Esperanza, muestran una relación muy significativa. Tabla 4. 


\section{TABLA No 5}

Relación entre peso fetal estimado por ecografía y peso del recién nacido

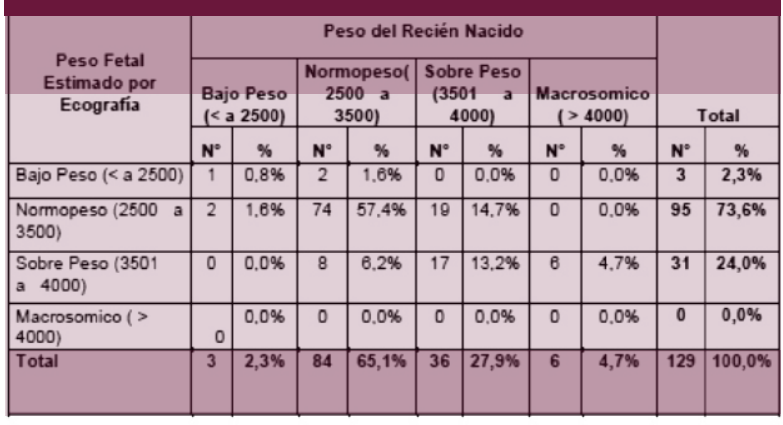

Fuente: Ficha de recolección de datos.

$$
\text { Chi=52,874 gl=6 P=0,000 }<\mathrm{a}=0,05
$$

\begin{tabular}{|l|r|r|r|}
\hline & \multicolumn{1}{|c|}{ Palor } & gl & \multicolumn{1}{|c|}{$\begin{array}{c}\text { Sig. asintótica } \\
\text { (bilateral) }\end{array}$} \\
\hline & $52,874^{\circ}$ & 6 & 0,000 \\
\hline Chi-cuadrado de Pearson & 44,150 & 6 & 0,000 \\
Razón de verosimilitudes & 39,671 & 1 & 0,000 \\
Asociación lineal por lineal & 129 & & \\
N de casos válidos & & & \\
\hline
\end{tabular}

a. 8 casillas $(66,7 \%)$ tienen una frecuencia esperada inferior a 5 . La frecuencia mínima esperada es, 07

Existe una alta coincidencia de los pesos estimados por ecografía con los pesos reales al nacimiento y al aplicar las pruebas de correlación de Pearson con un nivel de significancia del $5 \%$ tenemos que $\mathrm{Chi}=52,874$ $\mathrm{gl}=6 \mathrm{P}=0,000<\alpha=0,05$, quiere decir que existe una relación estadísticamente significativa entre el peso fetal estimado por ecografía y el peso al nacimiento. Tabla 5.

Al análisis correlación de todas las variables del estudio se observa $C h i=52,874(P=0,000)$ con ello se afirma que si existe relación estadísticamente significativo entre el peso estimado por ecografía y el peso del recién nacido en el Centro de Salud La Esperanza en el año 2016; por lo que rechazamos la hipótesis nula y aceptamos la hipótesis alterna.

\section{DISCUSIÓN}

La correcta estimación del peso fetal es un componente importante en la evaluación durante el trabajo de parto y el parto, porque sus alteraciones se asocian a aumento de la morbimortalidad fetal y condiciona la toma de decisiones con respecto a la vía del parto. El peso al nacer es reconocido como una variable importante que afecta la mortalidad perinatal.
Es por este motivo que la estimación de peso ecográfico tiene especial relevancia en la toma de decisiones clínicas tales como el momento de la interrupción y la vía de parto. La fórmula que mejor predice el peso fetal, determinado por ultrasonido, es la de Hadlock (1985), que evalúa tres parámetros; Diámetro Bi-parietal (DBP), Circunferencia Abdominal (CA) y Longitud de fémur (LF). (1)

Con respecto a las características gineco obstétricas de las gestantes, la edad materna predominante de las mujeres estuvo en el grupo etario de 18 a 29 años (64,3\%). La edad materna se asocia fuertemente con el posible daño del niño, muerte o enfermedad, nos permite cuantificar los grupos de riesgo, o sea aquellos grupos que tengan mayor posibilidad de daño (Alto Riesgo) o menor (Bajo Riesgo). A diferencia del estudio realizado por Fiestas y Col en 2003, quienes concluyen que la edad de las madres gestantes es de 26,9 +/- 6 años; así mismo Teva y Redondo en 2013 encontró que la media de edad materna fue de 31,78 años (DS $\pm 5,54$ años), siendo similar la edad de las gestantes estudiadas. $(13,19)$

Con respecto al peso fetal aproximado, determinado por ecografía, con mayor frecuencia fue el grupo de los normo pesos $(73,6 \%)$, seguido del sobrepeso $(24,0 \%)$ y solo el 2,3\% presentaron bajo peso. La determinación precisa del peso fetal es uno de los desafíos más importantes en la práctica gineco obstétrica diaria. $(4,5)$

Con respecto a la relación entre la edad gestacional estimado por ecografía y por fecha de última menstruación, en el estudio que se presenta, al realizar el análisis se evidencia relación significativa entre la edad gestacional estimada por ecografía y por fecha de última menstruación, con las pruebas de correlación de Pearson; se tiene que $\mathrm{Chi}=52,866$ y $\mathrm{P}=0,00000<$ $\alpha=0,05$. La determinación de la edad gestacional es el primer reto que se plantea y la ecografía es la herramienta fundamental que se emplea para este propósito. Además la ecografía resulta muy superior a la exploración clínica para determinar el correcto intervalo de crecimiento fetal. Sin embargo, desde la década de los noventa se han venido publicando diversos artículos que han informado que las estimaciones del peso fetal utilizando la fecha de última menstruación, o la palpación abdominal e inclusive la opinión de las madres tienen tanta exactitud como el ultrasonido para la predicción del peso fetal, con la ventaja de que son métodos económicos, inocuos y disponibles en cualquier momento. $(9,12)$ 
Con respecto a la relación entre peso fetal estimado por ecografía y peso del recién nacido, al realizar este análisis demostramos que sí existe relación significativa entre el peso fetal estimada por ecografía y el peso del recién nacido. Se puede observar que existe una alta coincidencia de los pesos estimados por ecografía con los pesos reales al nacimiento, y al aplicar las pruebas de correlación de Pearson con un nivel de significancia del $5 \%$ tenemos que $\mathrm{Chi}=52,874 \quad \mathrm{P}=$ $0.000<\alpha=0,05$. Sin embargo, en general podemos afirmar que el peso fetal estimado por ecografía si se relaciona significativamente con el peso del recién nacido en el estudio que presentamos. (15)

En comparación al estudio realizado por Manuelo, concluyó que los modelos que evalúan el peso fetal por ecografía subestiman el peso al nacer de los recién nacidos a término. El modelo que presenta menor error absoluto, menor error porcentual y mejor correlación positiva con el peso al nacer es el modelo de Hadlock. El cálculo o estimación del peso fetal en Obstetricia es muy importante, porque nos permite evaluar el tamaño fetal, estado nutricional del feto, trastornos del crecimiento. Además, en determinados casos es de vital importancia conocer el peso fetal para proyectarnos a las posibles intervenciones médicas. La relación peso fetal estimado con ecografía y peso del recién nacido, en los antecedentes es similar al encontrado en nuestra investigación lo que significa que el uso de la tecnología como la ecografía en embarazos a término es muy útil y un instrumento de apoyo para el diagnóstico y toma de decisiones correctas y oportunas con respecto al parto. Y con ello disminuir los riesgos de morbimortalidad materna e infantil. (20)

Se concluye que las características de las gestantes participantes en el estudio, en su mayoría presentaron edades entre los 18 a 29 años (64,3\%), con grado de instrucción secundaria completa $(63,6 \%)$, con talla promedio de $1,50 \mathrm{~cm}$ a $1,60 \mathrm{~cm}(62,8 \%)$, y peso materno promedio de 64 a $76 \mathrm{~kg}(66.6 \%)$, secundíparas $(38,8 \%)$, con edad gestacional por fecha de última menstruación de 39 y 40 semanas $(44,2 \%$ y $31.8 \%$ ) y por ecografía de 39 y 40 semanas ( $42,6 \%$ y $27,1 \%$ ) respectivamente.

El peso fetal aproximado determinado por ecografía con mayor frecuencia fue el grupo de los normo pesos $(73,6 \%)$, seguido del sobrepeso $(24 \%)$ y solo el $2,3 \%$ presentaron bajo peso. Así mismo el peso fetal calculado por fecha de ultima menstruación - Altura Uterina -Altura de presentación se tuvo normo pesos $(38,8 \%)$, sobrepesos $(38,0 \%)$ y el $15,5 \%$ bajo peso.
El peso real que presentaron los recién nacidos inmediatamente después del expulsivo fueron: Normo pesos de 2500 a $3500 \mathrm{gr}(65,1 \%)$, con sobrepeso entre 3501 a 4000 gr (27,9\%), macrosómicos con mayor de $4000 \mathrm{gr}(4,7 \%)$, y el $2,3 \%$ nació con bajo peso $<2500$ gr).

La correlación entre el peso fetal estimado por ecografía y peso del recién nacido en gestantes del Centro de Salud La Esperanza en el año 2016 es estadísticamente significativo, $(\mathrm{Chi}=52,874$ y $\mathrm{p}=$ $0,000)$.

Se recomienda que los obstetras asistenciales promuevan de manera rutinaria la realización de ecografías obstétricas y la estimación del peso fetal según la fórmula de Hadlock a todas las gestantes a término, por ser una ayuda diagnóstica efectiva y de vital importancia para decidir la vía y características del parto. Al MINSA promover y fortalecer las capacidades de los profesionales obstetras mediante cursos de capacitación en monitoreo electrónico fetal y diagnóstico por imágenes en obstetricia, de esa manera uniformizar criterios para la atención del parto diagnosticando precozmente los factores de riesgo y la toma de decisiones oportunas tendientes a evitar la morbimortalidad materna y fetal. A los gerentes de los centros de salud debido a la correspondencia de la validez de la ecografía para diagnosticar el peso fetal y su importancia dentro del desarrollo fetal, se sugiere a los directivos de la institución (Jefe del centro de salud), dirigir acciones con tendencia a estandarizar, protocolizar e implementar con un equipo ecográfico en sala de partos, y/o centros obstétricos, así mismo contar con obstetras capacitadas o especialistas en el tema de modo que se garantice la calidad dentro del control ecográfico.

Se recomienda en la comunidad, realizar actividades estratégicas de promoción y prevención en la población femenina a través de sesiones educativas y socio dramas, para aumentar la cobertura y captación precoz de la gestante; así determinar fecha de última menstruación exacta y confiable, que permita estimar el peso fetal oportunamente contrastándolo con la ecografía, y de este modo evitar posibles morbilidades fetales en el transcurso de la gestación e incluso en el parto. 


\section{REFERENCIAS BIBLIOGRÁFICAS}

1. Callen P. Mediciones utilizadas para evaluar el peso, el crecimiento y las proporciones corporales del feto, En: Callen, P. Ecografía en Obstetricia y Ginecologia. Edit Médica Panamericana; 4a edición; 2012:988-93

2. Vásquez C, Vásquez J, Febles J. Eficacia de la estimación del peso fetal por ultrasonido para la predicción del bajo peso al nacer. Rev Cubana Obstet Ginecol 2013; 10-13.

3. Pan American Health Organization (OPS) Organización Mundial de la Salud (OMS) [En línea]. Mayor acceso a la ecografía médica salvaría vidas maternas y neonatales en América Latina y el Caribe [Consultado el 27 de mayo de 2015]. Disponible en: http:/www.paho.org/hq/index.php?...11405\%3Aecografias... vidas...neonatales.

4. Shepard MJ, Ricards V, Berkowitz R. An evaluation of two equations for predicting fetal weight by ultrasound.Am J Obstet Gynecol 1982; 142: 47.

5. Gerard N. Estimación de peso fetal [Monografía en internet] Medicine Specialties Obstetricia y Ginecología General Obstetricia. [Citado 21 de abril 2015]. Disponible en: http://emedicine.medscape.com/obstetrics_gynecology.

6. Machado U. [En línea]. Estimación clínica y ultrasonográfica del peso fetal en embarazos a términos. 2013. [Consultado el 31 de mayo de 2015]. Disponible en:

http://www.elsevier.es/eop/s0210- 573x(12)00135-9.pdf Mazhar, D. [En línea]. Sultrasonic foetal weight estimation at term in bangladeshi womes-a comparative study of eigt formulae. 2011. 56 [Consultado el 31 de mayo de 2015]. Disponible en:

http://www.ncbi.nlm.nih.gov/pubmed/16530919

7. Pérez V, Carvajal J, Vera C. ¿Es la evaluación ultrasonográfica del peso fetal influida por la fórmula seleccionada? Rev. chil. obstet. ginecol. 2010; 75(2): 140-141.

8. Montesinos D. [En línea]. Factores de riesgo perinatales para peso bajo en recién nacidos a término del Hospital Gineco Obstetrico Isidro Ayora, Quito 2012. [Consultado el 31 de mayo de 2015]. Disponible en:

http://www.dspace.uce.edu.ec/bitstream/25000/1140/1/tuce-0006-38.pdf

9. Fescina R, De Mucio B, Díaz J, Martínez G, Abreu M, Camacho V, Schwarcz R. [Texto]. Salud Sexual y Reproductiva. Guías para la atención de la Mujer y el Recién Nacido Focalizadas en APS. Centro Latinoamericano de Perinatología Salud de la Mujer y Reproductiva CLAP/SMR-OPS/OMS 2007. № 1562 .

10.González J. [Texto]. Desarrollo y crecimiento del embrión y feto. En: González-Merlo J, Del Sol J.R, editores. Obstetricia. 4a edición, Barcelona: Masson, S.A; 2002. p 94-95.

11. Schwarcz R. Ultrasonido en obstetricia. En:Schwarcz, R. 7a ed. Argentina: Edit. El Ateneo. 2005:107-19.

12. Faneite P. Resultados perinatales en embarazos de alto riesgo.
Rev Obstet Ginecol Venez. 2001; 61 (1): 13-18 [consultado: 11 de agosto de 2015]

13. Díaz M, López J, García M, Herrera A, Meléndez M, Salas K. Cálculo de peso al nacer por ultrasonido en las embarazadas de alto riesgo. Salus, versión ISSN 1316-7138, Salus vol.15 no.3 Venezuela dic. 2011.[consultado: 11 de agosto de 2015]Disponible en:

http://www.scielo.org.ve/scielo.php?script=sci_arttext\&pid=S 1316-71382011000300006\&lang=pt

14. Teva M, Redondo R, Rodriguez I, Martinez C. Análisis de la tasa de detección de fetos macrosómicos mediante ecografía, Revista chilena de obstetricia y ginecología.versión On-line ISSN 0717-7526, Rev. chil. obstet. ginecol. vol.78 no.1 Santiago 2013, [consultado: 11 de agosto de 2015] Disponible en : http://dx.doi.org/10.4067/S0717-75262013000100003

15. Ferreiro R, Lemay A. Rev. Cubana Obstet Ginecol v.36 n.(4) Ciudad de La Habana oct.-dic. 2010. [ consultado: 11 de agosto de 2015]. Disponible en :

http://scielo.sld.cu/scielo.php?script= sci_arttext\&pid=S0138$600 \times 2010000400003$

16. Villafuerte C. Lima, Perú 2011. Propuesta de un nuevo puntaje para optimizar estimados ecográficos de peso fetal: Servicio de Obstetricia del Hospital Nacional Guillermo Almenara.

17. Anderson N, Jolley I, Jand. Wells J.E: Sonographic estimation of fetal weight: comparison of bias, precision and consistency using 12 different formulae, Ultrasound Obstet Gynecol 2007 ; $30: 173-179$

18. Rodríguez C, Quispe J. Revista peruana de ginecología y obstetricia versión on line ISSN 2304-5132 Rev. Perú. Ginecol. Obstet. vol.60. no (3). Lima jul./set .2014. [consultado: 13 de agosto de 2015] Disponible en:

http://www.scielo.org.pe/scielo.php?pid=S230451322014000300003\&script

19. Rojas J, Garay M, Ortiz C, Flores H, Huaroto F, Chico H, et al. Anales de la Facultad de Medicina versión impresa ISSN 10255583 An. Fac. med. v.70 n.(2) Lima jun. 2009. [consultado: 13 de agosto de 2015] Disponible en:

http://www.scielo.org.pe/scielo.php?script= sci_arttext\&pid=S1025-55832009000200005,

20. Fiestas C, Valera D, Palacios J, Gonzales L, Bardales B, Cisneros J, Ginecol obstet 2003; 49(4):214-218. [consultado: 13 de agosto de 2015]

Disponible:http://sisbib.unmsm.edu.pe/bvrevistas/ginecologi a/vol49_n4/a03.htm.

21. Manuelo B. (Tacna -Perú 2009) En su trabajo titulado, "Correlación entre el peso fetal estimado por ecografía según shepard, campbell y hadlock y el peso de los recién nacidos a término del hospital Hipólito Unanue de Tacna 2009".

\section{Correspondencia}

Luis Fredy Choque Mamani

luischoque_258@hotmail.com
Fecha de recepción: 10 de abril de 2018

Fecha de aceptación: 12 de junio de 2018 